\title{
REMARKS ON THE BORDISM ALGEBRA OF INVOLUTIONS
}

\author{
C. S. HOO
}

1. Introduction. Let $\Omega_{*}$ denote the unoriented bordism ring and let $R_{*}\left(Z_{2}\right)$ be the unoriented bordism group of involutions. Then $\mathfrak{R}_{*}\left(Z_{2}\right)$ is a module over $\mathfrak{R}_{*}$. In [1], it is shown that $\mathfrak{R}_{*}\left(Z_{2}\right)$ has $\left\{\left[A, S^{n}\right]\right\}_{n=0}^{\infty}$ as a basis over $\Re_{*}$, where $\left[A, S^{n}\right]$ is the bordism class of the antipodal involution on the $n$-sphere. Let $x_{n}=\left[A, S^{n}\right]$ $+\sum_{j=0}^{n-1}\left[P^{n-j}\right] x_{j}$ for each $n \geqq 0$. Then $\left\{x_{n}\right\}_{n=0}^{\infty}$ is also a basis for $R_{*}\left(Z_{2}\right)$ over $\Omega_{*}$. This has the advantage that $x_{n}$ belongs to the reduced group $\tilde{\Re}_{*}\left(Z_{2}\right)$ for $n \geqq 1$.

In [2], Su showed that $\mathbb{R}_{*}\left(Z_{2}\right)$ is a Hopf algebra over $\mathfrak{R}_{*}$, the multiplication being induced by the $H$-space multiplication of the classifying space $B\left(Z_{2}\right)$, and the comultiplication being induced by the diagonal map. We refer the reader to [1] and [2] for definitions and terminology.

It is natural then to ask for the multiplication law in $\mathfrak{R}_{*}\left(Z_{2}\right)$. In [2], Su showed that the multiplication satisfies $x_{m} x_{n}=(m, n) x_{m+n}$ $\bmod A_{m+n}$ where $(m, n)=(m+n) ! / m ! n !$ and $A_{m+n}$ is the $\mathbb{R}_{*}$ module generated by $x_{0}, x_{1}, \cdots, x_{m+n-1}$. In general, this congruence cannot be replaced by an actual equation. For example, by explicit computation, one can show that $x_{1} x_{2}=x_{3}+\left[P^{2}\right] x_{1}$. In this note, we show, however, that this congruence can be replaced by an equation if $m$ and $n$ are both odd. Precisely, we show

THEOREM.

$$
x_{2 m+1} x_{2 n+1}=0 \quad \text { for all } m, n .
$$

We are unable to give a complete description of the multiplication although we have some partial results.

2. In this section, $\left[A, S^{n}\right]$ will always denote the bordism class of the antipodal involution on the $n$-sphere. In connection with any bordism class $\left[T, M^{n}\right], c$ will always stand for the characteristic class of the involution. Stiefel Whitney classes of manifolds will always be denoted by $w_{i}$. Also, the letter $d$, with or without subscripts, will always denote the generator of $H^{1}\left(P^{n}, Z_{2}\right)$, the dimension $n$ of the projective space $P^{n}$ being clear from the context. Finally, homology and cohomology will always be taken with coefficients $\bmod 2$.

Received by the editors January 20, 1966. 
Lemma 1. $\left[A, S^{1}\right]\left[A, S^{n}\right]=0$ for odd $n$.

Proof. The characteristic class of the involution is given by

$$
c=d_{1} \otimes 1+1 \otimes d_{2} \text {. }
$$

Further, the Stiefel-Whitney classes of $P^{1} \times P^{n}$ are given by

$$
w_{i}=\left(\begin{array}{c}
n+1 \\
i
\end{array}\right) 1 \otimes d_{2}^{i}
$$

Now $\left[A, S^{1}\right]\left[A, S^{n}\right]$ is completely determined by its involution numbers

$$
\left\langle w_{i_{1}} \cdots w_{i_{r}}\left(P^{1} \times P^{n}\right) c^{l}, P^{1} \times P^{n}\right\rangle
$$

where $i_{1}+\cdots+i_{r}=n+1-l$. But

$$
c^{l}=\sum_{i=0}^{1}\left(\begin{array}{l}
l \\
i
\end{array}\right) d_{1}^{i} \otimes d_{2}^{l-i} .
$$

Hence the above involution number is

$$
\sum_{i=0}^{1}\left(\begin{array}{l}
l \\
\imath
\end{array}\right)\left\langle\prod_{j=1}^{r}\left(\begin{array}{c}
n+1 \\
i_{j}
\end{array}\right) d_{1}^{i_{j}} \otimes d_{2}^{n+1-i_{j}}, P^{1} \times P^{n}\right\rangle=l \prod_{j=1}^{r}\left(\begin{array}{c}
n+1 \\
i_{j}
\end{array}\right) .
$$

This is zero if $l$ is even. On the other hand, if $l$ is odd, then the sum $i_{1}+\cdots+i_{r}=n+1-l$ is odd since $n$ is odd. Hence some $i_{j}$ is odd. But this means that

$$
\left(\begin{array}{c}
n+1 \\
i_{j}
\end{array}\right)=0
$$

Thus all the involution numbers vanish and hence the lemma follows.

Note. Since $x_{1}=\left[A, S^{1}\right]$, we have then that $x_{1}\left[A, S^{2 n+1}\right]=0$ for all $n$.

LEMMA 2. $x_{1} x_{2 n+1}=0$ for all $n \geqq 0$.

Proof. If $n=0$, then $x_{1}^{2}=0$ by Proposition 3.4 of [2]. We now proceed by induction. Suppose that the result is true for all $j<n$. Now

$$
\begin{aligned}
x_{1} x_{2 n+1} & =x_{1}\left[A, S^{2 n+1}\right]+\sum_{j=0}^{2 n} x_{1} x_{j}\left[P^{2 n+1-j}\right] \\
& =\sum_{j=0}^{2 n} x_{1} x_{j}\left[P^{2 n+1-j}\right] \text { by Lemma } 1 .
\end{aligned}
$$


Since $\left[P^{2 n+1-j}\right]=0$ for even $j$, we have that

$$
x_{1} x_{2 n+1}=\sum_{j=0}^{n-1} x_{1} x_{2 j+1}\left[P^{2 n-2 j}\right] .
$$

By hypothesis $x_{1} x_{2 j+1}=0$ for all $j<n$. Hence $x_{1} x_{2 n+1}=0$.

LEMMA 3. $x_{1} x_{n}=0$ if and only if $n$ is odd.

Proof. If $n$ is odd, the result follows by Lemma 2 . It remains to show that if $x_{1} x_{n}=0$ then $n$ is odd, or equivalently that if $n$ is even then $x_{1} x_{n} \neq 0$. But by Su's result, we have that $x_{1} x_{n}=x_{n+1} \bmod A_{n+1}$ if $n$ is even. Hence $x_{1} x_{n} \neq 0$.

ThEOREM. $x_{2 n+1} \in x_{1} R_{*}\left(Z_{2}\right)$ for all $n$ and hence $x_{2 m+1} x_{2 n+1}=0$ for all $m, n$.

Proof. We first prove that $x_{2 n+1} \in x_{1} R_{*}\left(Z_{2}\right)$. Clearly this is true if $n=0$. We now proceed by induction. Suppose $x_{2 i+1} \in x_{1} \Re_{*}\left(Z_{2}\right)$ for all $i<n$. Now

$$
x_{1} x_{2 n}=x_{2 n+1}+\sum_{j=0}^{2 n} a_{2 n+1-j} x_{j}
$$

where $a_{2 n+1-j} \in \mathbb{R}_{2 n+1-j}$. Multiplying by $x_{1}$ and applying Lemma 2 we obtain the equation

$$
\sum_{j=0}^{2 n} a_{2 n+1-j} x_{1} x_{j}=0
$$

Again applying Lemma 2, we simplify the expression to obtain

$$
\sum_{j=0}^{n} a_{2 n+1-2 j} x_{1} x_{2 j}=0 .
$$

Writing this out in detail we obtain

$$
a_{1} x_{1} x_{2 n}+a_{3} x_{1} x_{2 n-2}+\cdots+a_{2 n-1} x_{1} x_{2}+a_{2 n+1} x_{1}=0 .
$$

Now we recall that $x_{1} x_{2 j}=x_{2 j+1}+y_{j}$ where $y_{j} \in A_{2 j+1}$. Hence we obtain the equation

$$
\begin{aligned}
a_{1} x_{2 n+1}+a_{1} y_{n}+a_{3} x_{2 n-1}+a_{3} y_{n-1} & +\cdots \\
& +a_{2 n-1} x_{3}+a_{2 n-1} y_{1}+a_{2 n+1} x_{1}=0 .
\end{aligned}
$$

Since the $x_{j}$ form a basis, we conclude that

$$
0=a_{1}=a_{3}=\cdots=a_{2 n-1}=a_{2 n+1} .
$$


Thus we now have

$$
x_{1} x_{2 n}=x_{2 n+1}+\sum_{j=0}^{n-1} a_{2 n-2 j} x_{2 j+1} .
$$

By hypothesis, each $x_{2 j+1} \in x_{1} \Re_{*}\left(Z_{2}\right)$ for $j \leqq n-1$. Hence $x_{2 n+1}$ $\in x_{1} R_{*}\left(Z_{2}\right)$. Finally, the last statement of the theorem follows from the fact that the multiplication is commutative and that $x_{1}^{2}=0$.

\section{REFERENCES}

1. P. E. Conner and E. E. Floyd, Differentiable periodic maps, Ergebnisse der Mathematik und ihrer Grenzgebiete, Vol. 33, Springer-Verlag, Berlin, 1964.

2. J. C. Su, $A$ note on the bordism algebra of involutions, Michigan Math. J. 12 (1965), 25-31.

University of Alberta, Edmonton 